\title{
Synchronicity: The Role of Midbrain Dopamine in Whole-Brain Coordination
}

\author{
(D) Jeff A. Beeler, ${ }^{1}$ and ${ }^{\circledR}$ Jakob Kisbye Dreyer ${ }^{2}$
}

https://doi.org/10.1523/ENEURO.0345-18.2019

${ }^{1}$ Queens College and the Graduate Center, City University New York, New York 11367, NY, ${ }^{2}$ Department of Neuroscience and Pharmacology, University of Copenhagen, Copenhagen 2200, Denmark

\begin{abstract}
Midbrain dopamine seems to play an outsized role in motivated behavior and learning. Widely associated with mediating reward-related behavior, decision making, and learning, dopamine continues to generate controversies in the field. While many studies and theories focus on what dopamine cells encode, the question of how the midbrain derives the information it encodes is poorly understood and comparatively less addressed. Recent anatomical studies suggest greater diversity and complexity of afferent inputs than previously appreciated, requiring rethinking of prior models. Here, we elaborate a hypothesis that construes midbrain dopamine as implementing a Bayesian selector in which individual dopamine cells sample afferent activity across distributed brain substrates, comprising evidence to be evaluated on the extent to which stimuli in the on-going sensorimotor stream organizes distributed, parallel processing, reflecting implicit value. To effectively generate a temporally resolved phasic signal, a population of dopamine cells must exhibit synchronous activity. We argue that synchronous activity across a population of dopamine cells signals consensus across distributed afferent substrates, invigorating responding to recognized opportunities and facilitating further learning. In framing our hypothesis, we shift from the question of how value is computed to the broader question of how the brain achieves coordination across distributed, parallel processing. We posit the midbrain is part of an "axis of agency" in which the prefrontal cortex (PFC), basal ganglia (BGS), and midbrain form an axis mediating control, coordination, and consensus, respectively.
\end{abstract}

Key words: coherence; dopamine; phasic dopamine; striatum; synchronous dopamine activity

\section{Significance Statement}

Dopamine is widely associated with providing value-related signals that serve activational and teaching functions. We shift focus from computing value-related signals and develop a hypothesis that suggest midbrain dopamine is sampling and integrating distributed neural activity to recognize and signal consensus across distributed, parallel processing. We posit each dopamine cell acts like an index of afferent activity and serves as a Bayesian unit detecting opportunity reflected in activity across distributed substrates. When cells across a dopamine population converge on the same temporal patterns, dopamine cell synchrony emerges generating a consensus signal that facilitates responding and learning. In this view, the fundamental role of dopamine is to signal consensus across parallel processing to facilitate unified behavioral responding.

\section{Introduction}

The midbrain dopamine system is integral to mediating value-based neural activity underlying adaptive behavior

Received August 31, 2018; accepted March 31, 2019; First published April 19, 2019.

The authors declare no competing financial interests.
(Wise, 2004; Schultz, 2007; Berridge, 2007; Salamone and Correa, 2012). Evidence continues to accumulate that midbrain dopamine can signal (1) obtained value, (2) value 
prediction, and (3) value prediction errors (Watabe-Uchida et al., 2017; Berke, 2018). Dopamine activity has been shown to be sufficient for mediating learned associations between stimuli and reward (Steinberg et al., 2013), demonstrating it can serve a crucial role as a value teaching signal (Schultz, 1998; Hart et al., 2014; Eshel et al., 2016; Kishida et al., 2016; Sharpe et al., 2017). Dopamine has been shown to modulate value-based behavioral choice and to increase (energize) responding to value predictive stimuli (Roitman, 2004; Hamid et al., 2016; Ko and Wanat, 2016; da Silva et al., 2018; Saunders et al., 2018). Dopamine has long been believed to provide a simple, scalar signal broadcast widely across the brain (Schultz, 1998; Bar-Gad et al., 2003; Joshua et al., 2009), essentially uniform across dopamine cells (Schultz, 1998; Eshel et al., 2016). Insofar as decision making is distributed across neural substrates, value must be mapped onto those distributed processes. By widely broadcasting a uniform, scalar signal, dopamine is commonly believed to deliver value information across neural substrates, mediating value-based modulation of processing and plasticity in target regions.

The nature of dopamine's contribution to value-based adaptive behavior continues to be contentious. While evidence demonstrates that dopamine can encode prediction errors (Hart et al., 2014; Eshel et al., 2015; WatabeUchida et al., 2017), dopamine can signal value per se as well, described by Hamid et al. (2016; Berke, 2018) as an instantaneous value signal. Dopamine signals are not restricted to reward. Bromberg-Martin has proposed that dopamine can provide alerting, reward and aversive signals (Bromberg-Martin et al., 2010), although these can be construed as value related. Dopamine responds to novelty (Horvitz, 2000; Overton et al., 2014), although this can be construed as an "information bonus" (Kakade and Dayan, 2002). Dopamine signals have been linked to motor activity independent of reward (Pasquereau and Turner, 2015; Dodson et al., 2016; Howe and Dombeck, 2016). Other evidence suggests that moment-to-moment motivational states can modulate dopamine (Satoh et al., 2003; Syed et al., 2016). Dopamine prediction error signals are intimately linked to timing of events (Pasquereau and Turner, 2015; Takahashi et al., 2016; Starkweather et al., 2017; Coddington and Dudman, 2018; Langdon et al., 2018), and conversely, changes in dopamine have been demonstrated to affect timing (Soares et al., 2016). Dopamine has been demonstrated to play a role in arousal (Eban-Rothschild et al., 2016), suggesting an additional dimension in dopamine signaling. The notion that dopamine cells provide a uniform, broadly distributed

Acknowledgements: We thank Jon Horvitz, Andy Delamater, Carolyn Pytte, and Jake Jordan for helpful feedback on earlier versions of this manuscript. We also thank Michael J. Frank and Saleem Nicola for helpful discussions.

Correspondence should be addressed to Jeff $A$. Beeler at jbeeler@qc.cuny.edu

https://doi.org/10.1523/ENEURO.0345-18.2019

Copyright (C) 2019 Beeler and Dreyer

This is an open-access article distributed under the terms of the Creative Commons Attribution 4.0 International license, which permits unrestricted use, distribution and reproduction in any medium provided that the original work is properly attributed. signal is increasingly contested (Willuhn et al., 2012; Roeper, 2013; Lammel et al., 2014; Sanchez-Catalan et al., 2014; Morales and Margolis, 2017; Saunders et al., 2018), with data supporting both homogeneity across dopamine cell activity (Eshel et al., 2016) and diversity in dopamine signals in target regions (Matsumoto and Hikosaka, 2009; Lerner et al., 2015; Parker et al., 2016). In computational models, dopamine signals have been construed to represent abstracted value information divorced from details of stimuli and actions, i.e., "model-free" (Montague et al., 1996). It is becoming increasingly clear, however, that dopamine encodes and responds to features of stimuli and reward (Keiflin et al., 2019; Takahashi et al., 2017) and can be "model-based" (Daw et al., 2011; Daw, 2012; Langdon et al., 2018).

Many excellent reviews discuss the various issues outlined (Schultz et al., 2017; Berke, 2018; Watabe-Uchida et al., 2017). Here, we will describe an alternative view of dopamine function, shifting from the contentious question of what dopamine encodes to the comparatively less examined question of how dopamine encodes the information contained in its signals. Our hypothesis will posit that instead of serving an informational function of distributing reward-related information per se, dopamine can be construed as delivering a signal that serves to coordinate distributed, parallel processing by providing a "consensus" signal. We briefly review key characteristics of the midbrain dopamine system relevant to our hypothesis before tackling the task at hand.

\section{Current Ideas on Derivation of Dopamine Value-Related Signals: The Problem of Anatomy}

How is the dopamine signal derived? Earlier models were based on variants of the algorithmic actor-critic model (Barto, 1995). These models presupposed dopamine to encode a prediction error and so the task was to determine how the quantities necessary for that computation (e.g., expected reward $V_{t}$, obtained reward $r_{t}$ ) were delivered to the midbrain. These models centered around the basal ganglia (BGS) connections with the midbrain (Houk et al., 1995; Brown et al., 1999; Contreras-Vidal and Schultz, 1999). While elegant, the models did not align with anatomy (Joel et al., 2002). Subsequent models incorporated additional anatomic features, such as the "spiraling" connectivity (Haber et al., 2000) of midbrain dopamine and striatal territories (Haruno and Kawato, 2006), a role for the pedunculopontine tegmental nucleus in value representations (Kawato and Samejima, 2007) and the pathway from striatal matrix projection neurons to dopamine cell dendrites in the substantia nigra reticulata (Tan and Bullock, 2008), or simply including more contributing regions (Vitay and Hamker, 2014). Hazy and colleagues built a model around substrates known to contribute to Pavlovian learning (Hazy et al., 2007, 2010). More recently, Eshel et al. (2015) proposed that GABAergic cells in the midbrain receive expected reward information that ramps up as reward approaches and drives local inhibition of dopamine cells, providing a simple subtractive mechanism for deriving a reward prediction error 
(RPE). Although elegant, the Eshel model begs the question of where the expected value signal in midbrain GABA cells is derived, effectively returning us to prior models with an added layer of complexity.

What every proposed model to date shares in common is the premise that discrete quantities, such as expected value and received reward, are computed in specific afferent regions and delivered to the midbrain to be used in computing value-related signals, typically construed as an RPE. However, this tidy compartmentalization and assignment of computational terms to discrete afferents is not supported by evidence. In recent years, a spate of elegant anatomic studies has reinvestigated the connectivity of midbrain dopamine using newer tracing methods, including tracing input/output relationships. In these studies, what stands out is the extent to which the inputs to the dopamine cells, regardless of their projection targets, arise widely from across the brain (Watabe-Uchida et al., 2012; Yetnikoff et al., 2014; Beier et al., 2015; Lerner et al., 2015; Carta et al., 2019). That is, it is the diversity and apparent non-specificity of afferent inputs that is most striking (Yetnikoff et al., 2014). How is this to be reconciled with the notion of discrete regions computing and delivering, for example, expected reward? What are the rest of the afferent inputs doing? A recent study by Tian et al. (2016) further highlights this problem. The authors recorded from different afferent inputs to the midbrain, as well as midbrain dopamine cells, and correlated firing patterns in afferent projections with task activities (cue, receiving reward, etc.). Rather than any clear segregation in afferent regions of specific quantities, reward, predicted value, error signals, they found that all of these quantities were distributed across the inputs tested with many regions and even individual cells showing mixed patterns of phasic activity (e.g., both cue and reward activation), seemingly belying any notion of tidy segregation of discrete quantities being computed in localized neural substrates and sent to the midbrain. It seems a little like everything is everywhere.

Aside from the diversity of inputs and the promiscuity of value-related afferent information, there is a more fundamental problem facing models that propose discrete quantities are computed in specific afferent regions and delivered to the midbrain. Most of the regions contributing afferents to the midbrain operate with ensemble encoding; that is, information is differentially encoded in various patterns of neural activity. Dopamine neurons, in contrast, are believed to act largely en masse and collectively encode a value-related signal delivered as a uniform scalar. How is a representation of a stimulus or action encoded in a patterned subset of cells (a CS+, for example) in a region such as the amygdala translated into a single quantity (e.g., expected value) and then distributed broadly across a population of dopamine cells? Short of every neuron in an ensemble within a particular afferent region being connected with every dopamine cell, it is not clear how this ensemble->scalar translation could be achieved and uniformly deliver the required quantity across the midbrain.
Together, these issues argue that the notion that discrete quantities are computed in particular afferent regions and delivered to the midbrain is not consistent with the anatomy and largely untenable. Instead, it appears that the midbrain is integrating a highly diverse set of inputs drawn broadly from across the brain and that these inputs may contain various, non-segregated value-related information. While this provokes the question of what information is being integrated, how and to what end, before considering this we have to consider the particularities of the output side of the dopamine system.

\section{The Necessity of Dopamine Cell Cooperation: The Problem of Synchrony}

The midbrain dopamine nuclei contain a modest number of neurons, in rodents $\sim 20,000-40,000$ tyrosine hydroxylase expressing neurons, estimated to be between 400,000 and 600,000 in humans (Björklund and Dunnett, 2007). Each neuron projects extensively across a large territory of target region(s). Individual SNC dopamine neurons are estimated to make $100,000-250,000$ synapses in the rat and 1-2 million in humans (Bolam and Pissadaki, 2012), cover $6 \%$ of striatal volume and affect $\sim 75,000$ medium spiny neurons (MSNs) (Matsuda et al., 2009). Conversely, each MSN synapses with a few hundred to over a thousand dopamine terminals (Arbuthnott and Wickens, 2007; Bolam and Pissadaki, 2012) and each MSN is estimated to be under the influence of 100-200 different dopamine neurons (Matsuda et al., 2009). This broad axonal distribution with overlapping spheres of influence is characteristic of volume transmission attributed to dopamine (Agnati et al., 1995; Zoli et al., 1998). Rather than releasing neurotransmitter discretely in a synapse, which then transmits an ultra-targeted intra-synaptic signal quickly terminated by reuptake, dopamine operates by modulating its extracellular concentration (Garris et al., 1994; Gonon, 1997; Cragg and Rice, 2004; Moss and Bolam, 2008; Dreyer et al., 2010). These characteristics of midbrain dopamine suggest that to effectively transmit a signal, dopamine cells must work cooperatively.

A dopamine signal encoded through modulation of cell spiking is decoded in target regions by modulation in receptor occupancy: thus, encoding-decoding fundamentally reflects a transformation from spike rate (frequency modulation) to fluctuations in extracellular dopamine concentration (amplitude modulation). While many factors can modulate this transformation, the critical determinant is the degree of synchrony among spiking neurons relative to the clearance rate of the dopamine transporter (Dreyer et al., 2010; Dreyer and Hounsgaard, 2013; Dreyer, 2014). When spike activity is correlated between neurons on a timescale similar to $K_{\mathrm{m}} / V_{\max }$ (corresponding to $100 \mathrm{~ms}$ in nucleus accumbens and $40 \mathrm{~ms}$ in dorsal striatum), release will integrate and generate temporally resolved peaks and troughs in extracellular dopamine that maximize information transfer to postsynaptic targets. When spike activity is asynchronous, including asynchronous bursting activity, dopamine concentration will be distributed across time, reflecting the average spike rate. We will refer to "tonic" and "phasic" in terms of whether population spiking activity generates tem- 
porally resolved fluctuations in extracellular dopamine (phasic, in phase) or a distributed averaging of activity (tonic, dopamine tone). Rather than reflecting two distinct modes of signaling, tonic and phasic represent two ends of a spectrum in temporal partitioning of dopamine cell activity. Phasic dopamine signaling has been construed as arising from uniformity in bursting activity, consistent with electrophysiological evidence where dopamine cell recordings show a high percentage of tested cells exhibit similar and consistent responses to stimuli (Eshel et al., 2016), as well as with electrochemistry recordings of released dopamine, which fundamentally reflect the combined activity of hundreds of terminals (Dreyer et al., 2016).

While initially it was thought that dopamine cells are in some way coupled generating obligate synchrony, accumulating evidence favor emergent synchrony in which cells function autonomously, but independently come to generate the same temporal patterns of activity. Gap junctions between dopamine cells (Grace and Bunney, 1983) have been proposed to mediate uniformity in dopamine cell activity. These direct cell to cell connections may be less prevalent than originally thought, with estimates suggesting only $\sim 20-25 \%$ of dopamine cells are connected by gap junctions (Vandecasteele, 2005). These connections may provide a low-pass filter such that the activity in one neuron can influence firing rate in a connected neuron, but without transmitting yoked action potentials; no spike synchrony was observed in recorded pairs and mutual information was weak (Vandecasteele, 2005). There is little evidence that these gap junction connections induce an obligate synchrony, although such gap junctions could entrain network activity (Komendantov and Canavier, 2002) and may play a role in promoting emergent synchrony.

In studies examining pairs of dopamine cells, roughly $25 \%$ of dopamine cell pairs show significant correlation in spiking rate (Wilson et al., 1977; Hyland et al., 2002; Morris et al., 2004; Li et al., 2011) with little evidence of direct spike-tospike correlation (Morris et al., 2004). The degree of correlation can be modulated by reward associated stimuli, learning and pharmacological manipulations. Using noise correlation analysis (correlation between two cells in their trial-to-trial variation from average response), Joshua et al. (2009) demonstrate that correlation in spike variability between dopamine cells increases with salient stimuli (cue, outcome). Kim et al. (2012) show an increase from 34\% (noise) correlated cell pairs on initial exposure to a task to $49 \%$ and $66 \%$ after eight and 16 weeks of training, respectively. Finally, Li et al. (2011) demonstrated that application of nicotine increase the percentage of correlated dopamine cell pairs from $21 \%$ to $44 \%$. These data suggest emergent rather than obligate synchrony. Many have previously suggested that synchronous dopamine cell bursting activity is necessary to generate temporally resolved extracellular dopamine signals in target regions (Venton et al., 2003; Arbuthnott and Wickens, 2007; Dreyer et al., 2010; Owesson-White et al., 2012), an idea we will place at the center of our hypothesis.

\section{Shifting Frameworks: From Value to Coordination of Distributed, Parallel Processing}

We are presented then with a stark contrast between midbrain dopamine input, highly diverse afferent inputs drawn broadly from across brain regions in which valuerelated information does not appear to be neatly compartmentalized by region but rather widely distributed and intermixed, and the dopamine output system, which relies on relative uniformity of spiking activity across dopamine cell populations to transmit a simple scalar to target regions. It is this transformation from heterogeneity of input to homogeneity of output that will concern us: we propose the transformation from polyglot to monosyllable is fundamental to dopamine's function within the brain, and that the emergence of dopamine cell synchrony is the core mechanism mediating this function.

Before describing our hypothesis, we want to tentatively reframe the putative problem the dopamine system evolved to solve. Widely viewed as the "reward transmitter," various theories of dopamine describe a system that, in one way or another, helps the organism recognize and act on opportunities for value. In its most simplistic terms, the core evolutionary problem addressed is helping an organism to engage in actions that are advantageous, specifically by signaling value in some form (e.g., value per se, errors in value prediction).

We wish to consider dopamine in the context of a different core evolutionary problem. A fundamental question in neuroscience is how distributed, parallel processes are integrated into a functional "whole-brain" model to generate unitary organismal action (Breakspear, 2017; Christophel et al., 2017). The brain must process a continuous stream of on-going sensory information and from this parse actionable stimuli and emit advantageous responses, and do so rapidly (Cisek and Kalaska, 2010). Distributed, parallel processing facilitates rapid, efficient responding in the face of computational complexity. Each region/substrate forms its own, partial model of self-inworld. Simplistically, the hippocampus forms a spatial model: where actionable stimuli and events are located (Gauthier and Tank, 2018; Mamad et al., 2017; Stachenfeld et al., 2017). The amygdala forms a model of the valence of stimuli in relation to the organism (Beyeler et al., 2018; O'Neill et al., 2018; Pryce, 2018). The parietal cortex forms a model of egocentric sensorimotor space (Buneo and Andersen, 2006; Cui, 2014; Whitlock, 2014; Takiyama, 2015) and so on. Given that there is only one motor plant, these parallel models or representations must somehow be integrated into decision making that determines unitary organismal behavior, an organism cannot go right and left at the same time. Commonly construed as a simple scalar distributing value-related information, here we will entertain the possibility that dopamine provides a signal that coordinates rather than informs distributed, parallel processing, facilitating unitary self-in-world action from a multiplicity of neural representational models. 


\section{Searching for the Ghost in the Machine: A Case for Implicit Value}

The function broadly associated with dopamine involves recognizing available reward opportunities, weighting decision making to act on opportunity, energizing responding and facilitating learning about the context and predictors in which the reward occurred: in short, to facilitate adaptive choices in the face of opportunity. Is this not what the entire brain evolved to do? "Value" is a (perhaps the) fundamental organizing principle of the brain. It is not merely that value is reflected in on-going neural processing broadly across neural substrates (e.g., primary visual cortex, hippocampus) but that the brain is structurally shaped and modified by value. During early development, synapses are pruned to select those that yield functionality (i.e., value) to the organism. Throughout life, neural plasticity shapes circuits and weights synapses to benefit the organism, i.e., obtain value.

The challenge is ascertaining the root of the evaluative mechanisms that guide this value organization. How does the brain know what is good for it? While there are many potential answers to this question, one common answer is that dopamine distributes value information. Indeed, that is the core role of the reward transmitter regardless of one's view on the form in which this information is delivered. But this immediately begs the question "how does midbrain dopamine 'know'?" Who teaches the teacher? That is, how is this signal derived? All extant theories suggest that the midbrain obtains value information from afferent regions, but this quickly becomes circular. If dopamine is the teacher, how do afferent regions learn about value to inform the midbrain, and if afferent regions already recognize value, what need is there for a teaching signal in the first place? In short, the notion that dopamine distributes value information across the brain begs the question of how and where value is originally determined. Where is the ghost in the machine?

While the brain must select stimuli to which to direct its attention and generate advantageous responses, the most fundamental selection process is how the brain represents self and world in the first place. That is, it is neural activity itself that must be selected to create adaptive, productive self-in-world models that, in turn, yield adaptive behavior. This assertion does not resolve the question posed above about how value is determined, but simply restates it in neural terms. However, doing so provides a different perspective. Rather than construe value as some quantity computed somewhere, we view the brain as being in a constant process of selecting productive and diminishing non-productive neural activity. While we cannot pretend to explain how this happens, we do not know where the "ghost" resides, we can draw a tentative corollary: value is implicit in neural activity. While this may sound radical, it is consistent not only with a broad construal of the fundamental function of the brain in mediating adaptive behavior, it is consistent with empirical studies that tend to find value information, in a variety of forms, distributed broadly across the entire brain (e.g., Tian et al., 2016, but many others). From here we will sketch out our hypothesis of midbrain dopamine function, building around the core ideas that dopamine is (1) integrating diverse afferent activity that reflects implicit value and (2) generating a scalar output signal when this value information from different afferents temporally converges signaling "agreement," or consensus across distributed, parallel processing, that something important is happening now; that is, the whole brain is in agreement, here is an opportunity, time to act.

\section{Reconceptualizing Midbrain Dopamine as Sampling-Distributed Activity across the Brain}

Watabe-Uchida et al. (2012) note that afferents into the midbrain, rather than reflecting distinct connectivity between functional brain regions seems to arise in diagonal bands across the brain that do not respect functional regions. Rather than try to find order in afferent connectivity to the midbrain, we propose the midbrain randomly samples activity broadly across the brain and functions as an index of that activity, much in the way that a sample of stocks in the stock market serves as an index of the market. Consider the axons streaming from each afferent region to the midbrain as a vector where each axon delivering a continuous, rate-based signal is an element in the vector and the vector represents the total output to the midbrain from that afferent region. We posit that these axons, each an element in the afferent vector, are distributed randomly to dopamine neurons (Fig. $1 A$ ). If each dopamine neuron is, in turn, viewed as a vector composed of its various inputs, each dopamine cell represents a random sample of afferent activity where the afferent vectors from input regions collectively comprise a multidimensional space (Fig. 1B). We construe this space as event driven, i.e., that neural activity is continuous and constantly responding to on-going sensorimotor information. An event such as a cue-light, then, would be represented in this multidimensional space as a composite of the vectors representing individual afferent regions. Stimuli, actions, events, memories, everything encoded by the brain, would generate a unique composite in this multidimensional space.

Each individual dopamine cell represents a random sample of this multidimensional space. In inferential statistics, we use probability theory to ask "how likely is it that this difference arose as chance and if I repeated the study over and over would this effect persist?" An alternative strategy, although impractical, would be simply to simultaneously replicate a study thousands or millions of times always with a new randomly drawn sample. So, we argue, with dopamine. A single dopamine cell may increase its firing in response to increased afferent activity, but this could arise spuriously from the particular randomly distributed afferents it receives without reflecting something important arising in the multidimensional input space overall, such as a cue-light that comes to organize neural activity across multiple brain regions. Fortuitously, when a single dopamine cell starts bursting independent of the population, it will have little effect on output at target regions. Indeed, phasic activity is not a rare event correlated only with value. Dopamine cells continuously 


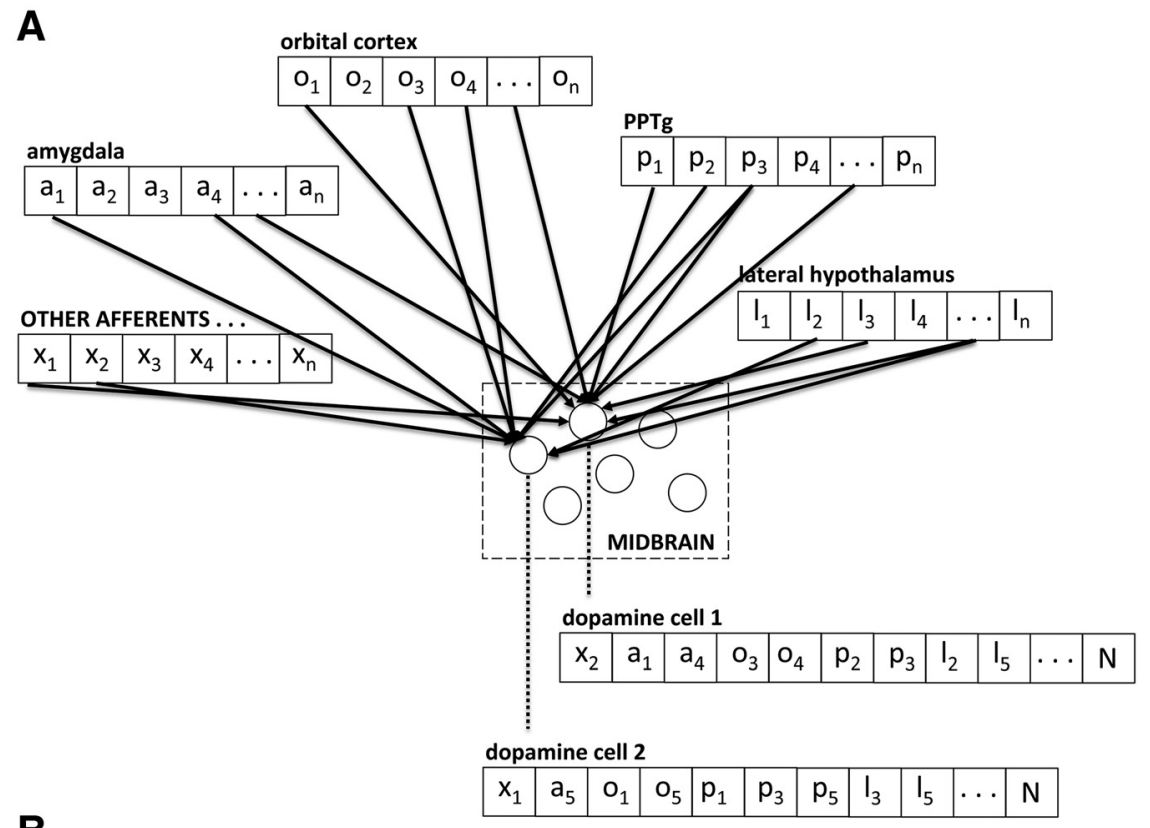

AFFERENT INPUT SPACE

\begin{tabular}{|l|l|l|l|l|l|}
\hline $\mathrm{o}_{1}$ & $\mathrm{o}_{2}$ & $\mathrm{o}_{3}$ & $\mathrm{o}_{4}$ & $\ldots$ & $\mathrm{o}_{\mathrm{n}}$ \\
\hline $\mathrm{a}_{1}$ & $\mathrm{a}_{2}$ & $\mathrm{a}_{3}$ & $\mathrm{a}_{4}$ & $\ldots$ & $\mathrm{a}_{\mathrm{n}}$ \\
\hline $\mathrm{p}_{1}$ & $\mathrm{p}_{2}$ & $\mathrm{p}_{3}$ & $\mathrm{p}_{4}$ & $\ldots$ & $\mathrm{p}_{\mathrm{n}}$ \\
\hline $\mathrm{I}_{1}$ & $\mathrm{I}_{2}$ & $\mathrm{l}_{3}$ & $\mathrm{I}_{4}$ & $\ldots$ & $\mathrm{l}_{\mathrm{n}}$ \\
\hline \multicolumn{7}{|c}{$\bullet$} \\
\hline $\mathrm{x}_{1}$ & $\mathrm{x}_{2}$ & $\mathrm{x}_{3}$ & $\mathrm{x}_{4}$ & $\ldots$ & $\mathrm{x}_{\mathrm{n}}$ \\
\hline
\end{tabular}

DOPAMINE CELL AFFERENT SAMPLING

\begin{tabular}{|c|c|c|c|c|c|c|c|c|c|c|}
\hline$x_{2}$ & $a_{1}$ & $a_{4}$ & $\mathrm{O}_{3}$ & $\mathrm{O}_{4}$ & $p_{2}$ & $p_{3}$ & $\mathrm{I}_{2}$ & $\mathrm{I}_{5}$ & $\cdots$ & $\mathrm{N}$ \\
\hline$x_{1}$ & $a_{5}$ & $\mathrm{O}_{1}$ & $\mathrm{O}_{5}$ & $p_{1}$ & $p_{3}$ & $p_{5}$ & $\mathrm{I}_{3}$ & $I_{5}$ & $\ldots$ & $\mathrm{N}$ \\
\hline $\mathrm{I}_{3}$ & $p_{5}$ & $\mathrm{O}_{4}$ & $\mathrm{O}_{9}$ & $a_{8}$ & $a_{4}$ & $x_{5}$ & $x_{3}$ & $\mathrm{I}_{2}$ & $\ldots$ & $\mathrm{N}$ \\
\hline$p_{7}$ & $a_{3}$ & $\mathrm{O}_{8}$ & $\mathrm{O}_{2}$ & $x_{9}$ & $x_{7}$ & $a_{6}$ & $\mathrm{I}_{9}$ & $\mathrm{I}_{4}$ & $\ldots$ & $\mathrm{N}$ \\
\hline \multicolumn{11}{|c|}{ • $\bullet \bullet$} \\
\hline$s_{5}$ & $\mathrm{~S}_{2}$ & $\mathrm{~s}_{1}$ & $\mathrm{~S}_{4}$ & $s_{9}$ & $s_{7}$ & $\mathrm{~S}_{6}$ & $\mathrm{~S}_{3}$ & $S_{8}$ & $\ldots$ & $\mathrm{N}$ \\
\hline
\end{tabular}

Figure 1. Random distribution of afferents to dopamine cells as sampling the input space. $\boldsymbol{A}$, Projections from each afferent region construed as a vector randomly distributed to individual dopamine cells. $\boldsymbol{B}$, Afferent projections to midbrain comprise a total vectorized input space from which individual dopamine cells represent random samples of that space.

intermix tonic and phasic activity (Marinelli and McCutcheon, 2014) with dopamine output determined by population activity (Dreyer et al., 2010); that is, when they fire synchronously. Thus, the 40,000 dopamine cells in the rat midbrain could be construed as 40,000 random samples, 40,000 "studies" occurring concurrently, and when a large percentage of them are driven to burst firing simultaneously, this generates a temporally resolved dopamine signal in target regions that carries value-related information. Because this multi-dimensional afferent input space is comprised of activity arising in parallel, distributed processing, for a stimulus such as a cue-light to generate population-based, synchronous phasic activity in midbrain dopamine, the stimulus must presumably have a widespread effect across neural regions. Because of this, we construe the emergence of temporally resolved phasic dopamine signals that arise from synchronous bursting activity as signaling consensus, a consensus across afferent regions about the importance of on-going sensorimotor information being processed in parallel across distributed substrates.

\section{Axis of Agency: Sketching a Broader Hypothesis of Coordination across Distributed Processing}

Our purpose here is to initially formulate a novel hypothesis of dopamine function. Because we shift our framework from the problem of computing and delivering value information to the problem of facilitating coordination across distributed, parallel processing, we must necessarily put our discussion of dopamine into this broader context and provide a rough sketch of how the brain achieves coordinated distributed processing, in which we posit the midbrain plays a specific role.

We posit four levels of substrate in describing coordination across distributed processing (Fig. 2). We posit four levels of substrate in describing coordination across distributed processing. The first is primary processing in individual neural regions, such as the amygdala, hippocampus and so on. Although these are intricately interconnected (e.g., frontal and parietal regions), each region specializes in capturing some aspect of an overall model of self-in-world. A second substrate level would be where 
disparate processing converges onto a substrate that does not have its own assigned task but is assigned to ensure that the work of the various parallel processing does not begin to conflict or go in different directions viewed collectively as a whole. We posit that the BGS serve this function (elaborated below), which we describe as integrative discriminative selection. "Integrative" because they integrate disparate inputs from across distributed processing and "discriminative selection" because, in turn, they modulate efferent targets (generally the same regions providing afferents) by selecting which activities to facilitate and which to inhibit (selection), doing so by discriminating which afferent inputs facilitate positive outcomes and which do not. This substrate can effectively modulate the activity of individual primary substrates "in the context" of other primary substrates: integrative discriminative selection. Third, we posit a signal that indicates when there is agreement across distributed processing to mobilize action and facilitate learning, a function we attribute to midbrain dopamine. We would describe this function as integrative consensus: integrative because multiple, diverse inputs are being integrated in a polling manner ("sampled") and consensus because the goal is to determine when something appears in the on-going sensorimotor stream that is recognized across multiple substrates as being of value and broadcasting a signal when this occurs. Finally, there needs to be some primary control structure that steers this "ship of coordination," which we attribute to the prefrontal cortex (PFC).

We will briefly describe this view of the BGS as it is central to elaborating our hypothesis of dopamine. It is difficult to cogently discuss the function of either dopamine or the BGS without taking both in account as they are densely interconnected (Watabe-Uchida et al., 2012; Guo et al., 2015) and their respective functions profoundly intertwined. We will not discuss primary processing or associated interconnectivity nor will we elaborate on the role of the PFC as "steering the ship" as much has already been written on the PFC as an executive locus.

\section{The BGS: Selection of Composite Representations}

The primary input nucleus of the BGS, the striatum, has been thought to be an integrative substrate for decades (Hikosaka et al., 2014; Kemp and Powell, 1971). Processing convergent information from multiple sources, the BGS in turn modulate the regions that provide afferent input, illustrated best by the cortico-BGS-cortical loops. Considered in the context of facilitating coordination across distributed, parallel processing, we propose that the BGS function analogous to a hidden layer in neural networks. Inputs are distributed across units in a combinatorial fashion allowing the discriminative selection of those combinatorial units associated with positive outcomes. Across learning, this combinatorial selection process progressively modulates those same brain regions that provide afferent input. Because the striatum is integrating information from across distributed, parallel processing into these combinatorial units, this provides a substrate in which activity in one region can be modulated "in the context of" other regions. We elaborate this briefly.

\section{The mosaic: substrate for combinatorial integration and selection}

Da Cunha et al. (2009) liken the striatum to a "mosaic of broken mirrors." This is to say that cortical representations are not mirrored directly in the striatum but are broken up, with fragments of cortical representations distributed broadly across striatal MSNs. Each MSNs, in turn, receives fragments of cortical representations in a combinatorial manner. Thus, discrete cortical representations become fragmented and intermixed: mosaic of broken mirrors. This is consistent with known anatomy. A single MSNs receives $\sim 5000$ cortical afferents (Alexander, 1994), although individual cortical axons will make only one to two contacts with a single MSNs (Tepper et al., 2007), suggesting both tremendous distribution and convergence. Conversely, a single cortical axon can innervate up to $14 \%$ of the striatum (Zheng and Wilson, 2002). While the striatum has been associated with dimension reduction (Bar-Gad et al., 2003), this fragmentation and expansion of cortical representations into combinatorial units (the mosaic) could be view as dimension enhancement. Introducing combinatorial dimensions allows a more expansive and powerful substrate for discriminative selection compared to faithfully recapitulating or mirroring cortical representations discretely in the striatum.

\section{At the crossroads: integrating distributed, parallel processing in selection}

Noting that the inputs from multiple afferent regions (amygdala, hippocampus, PFC) intersect in the ventral striatum, Humphries and Prescott (2010) characterized this intersection as a "crossroads" where the activity from disparate regions come together and interact, integrating their activities in the output of the ventral striatum. This convergence of afferents from disparate regions is observed across the striatum (Choi et al., 2017). Building on the mosaic idea above of expanded, fragmented and distributed cortical representations, the crossroads notion provides a basis whereby the selection and modulation of these fragmented cortical representations can be modulated by inputs from other regions, providing a substrate where distributed parallel processing across multiple afferent regions are integrated into a combinatorial "mosaic" in which what is being selected is not discrete actions or stimuli per se, but combinations of neural activity across distributed substrates that reflect parallel processing of stimuli and actions: a composite representation. In essence, the BGS select when concurrent activity across distributed substrates is productive and facilitates that concurrent activity in target/afferent regions.

\section{A little bit of everything, everywhere}

While most brain regions have been associated with particular kinds of information, the striatum exhibits remarkable malleability in its encoding. In virtually every task in which striatal activity has been monitored, every 


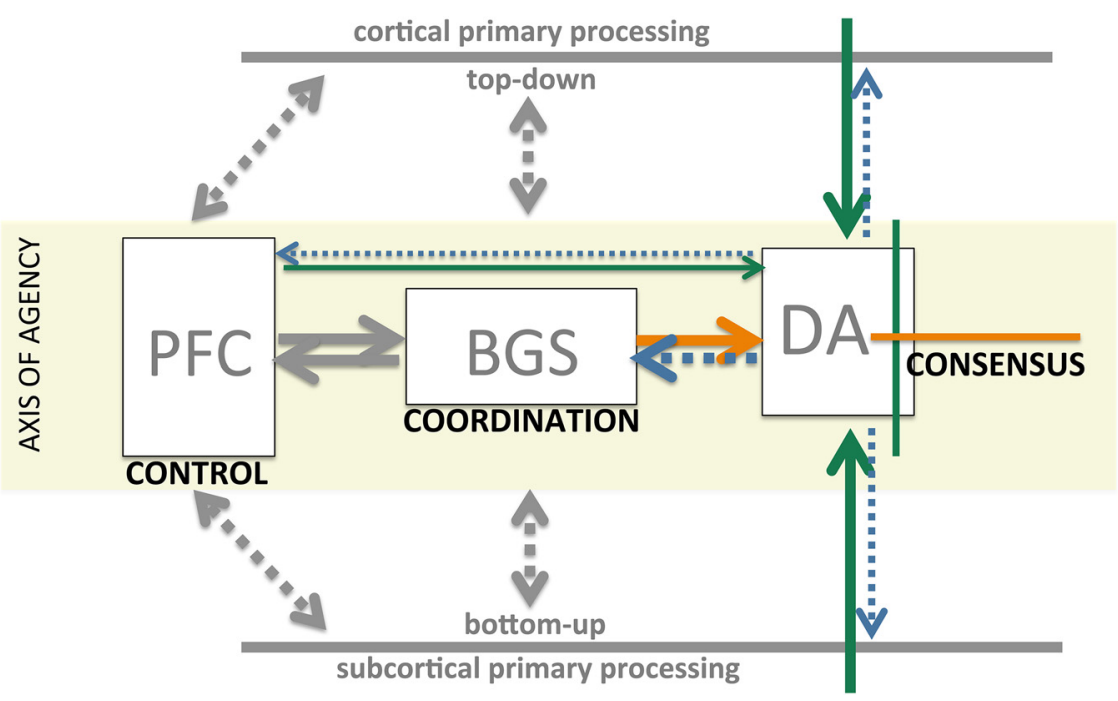

Figure 2. Schematic of "axis of agency." Primary processing substrates (distributed, parallel processing) represented abstractly in gray as either top-down or bottom-up substrates with reciprocal connections to the prefrontal cortex (PFC) and basal ganglia system (BGS), not detailed here. The three conceptual nodes in the axis of agency are indicated in boxes with their hypothetical role in mediating coordinated activity across distributed substrates noted in bold below. Only the inputs/outputs of dopamine, the focus of this perspective, are colored, with green and orange representing excitatory and inhibitory inputs and blue dopamine outputs. The role of the BGS and midbrain dopamine is elaborated below.

aspect of the task has been represented: stimuli/cues, actions, rewards as well as putative predicted value associated with these representations. Moreover, mediumspiny neurons will come to represent whatever is relevant in a task. If spatial locations are relevant, spatial locations will be represented in phasic MSN activity (Shibata et al., 2001; Chang et al., 2002). If time is important, time will be represented (Taha et al., 2007; Day et al., 2011). In short, across learning the striatum appears to form a representation of any task where salient aspects of the task are differentially represented in phasic activity in subsets of MSNs. This malleability is consistent with the notion of discriminative selection on a combinatorial substrate integrating disparate afferents, as we propose.

\section{Different kinds of integration}

In our characterization of the BGS and midbrain dopamine, we suggest both are integrating disparate afferents drawn broadly from across the brain, but the function of this integration is essentially opposite. During learning, dopamine cell activity becomes increasingly synchronous and homogeneous across a population of dopamine cells (Eshel et al., 2016). In contrast, MSNs develop differential patterns of phasic activity in response to discrete stimuli and task events (Tremblay et al., 1998; Hikosaka et al., 1989a, b,c; Nicola et al., 2004; Day et al., 2006; German and Fields, 2007), suggesting discriminative learning. In short, while dopamine cells in a population converge in their signaling, striatal cells diverge, facilitating a discriminative selection process.

\section{Striatal composite representation modulates distributed processing}

Gerraty et al. (2018) demonstrate that learning to associate a visual cue with reward involves dynamic changes in the coupling between striatum and multiple brain re- gions, including frontal and visual cortices, and that these changes are correlated with learning rate (see also den Ouden et al., 2010; van Schouwenburg et al., 2010; Horga et al., 2015). Thus, returning to our larger perspective of assigning the BGS a role in mediating coordinated activity across distributed, parallel processing, we suggest that the BGS, via the striatum, create a mosaic or combinatorial composite of activity from multiple, disparate neural regions (integrative) and use this composite substrate to discriminatively select those combinatorial units (analogous to hidden units) that are associated with positive outcomes to broadly modulate distributed processing across the brain, facilitating coordination across distributed processing that is advantageous to the organism. A critical aspect of this function is that it operates via disinhibition; that is, the BGS exert a tonic inhibition that is selectively released, an operational characteristic we will further build on below. A central requirement for this proposed striatal/BGS function is some mechanism by which selection is guided, a teaching signal. Dopamine has long been believed to provide this teaching signal (Schultz et al., 1997; Schultz, 1998; Wise, 2004), returning us to midbrain dopamine.

\section{Midbrain Consensus Signaling: Emergent Synchrony in Dopamine Cell Activity as a Bayesian Selector}

We propose that midbrain dopamine functions at the intersection of two axes (Fig. 3): (1) a largely excitatory axis "sampling" distributed, parallel activity that drives dopamine activity, particularly bursting and (2) an inhibitory axis derived from the striatum that gates dopamine cells as a function of striatal integrative discriminative selection process we described above. We propose that these two axes can be construed as "advocate" and 
A

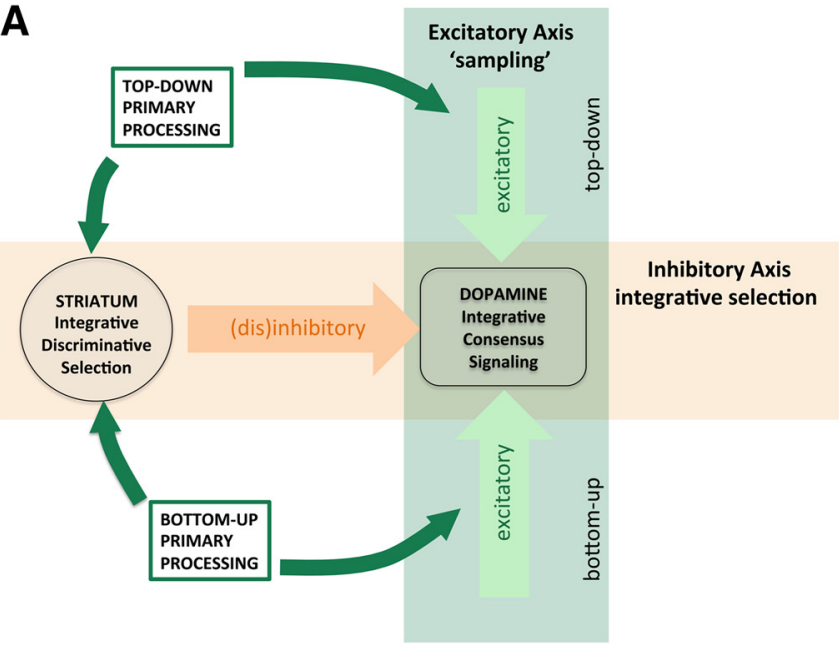

B

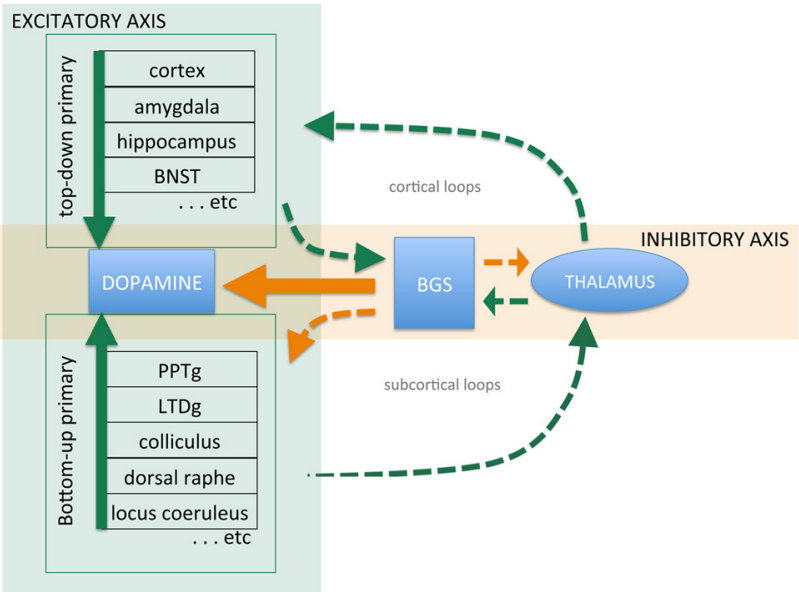

Figure 3. Two axes model of dopamine integrative consensus signaling. $\boldsymbol{A}$, Overall conceptual rendering of proposed model where midbrain dopamine integrates two primary axes of input, (1) a (dis)inhibitory axis arising from the BGS (ventral pallidum, striosomes, accumbens) and (2) a largely excitatory axis arising from distributed afferents across the brain, reflecting both top-down (e.g., cortical inputs, amygdala, hippocampus, BNST) and bottom-up information processing (e.g., collicular, multiple brainstem afferents). B, A more anatomic rendering incorporating cortical and subcortical loops through the BGS. For detailed cataloging of dopamine inputs, see Watabe-Uchida et al. (2012), Lerner et al. (2015), and Beier et al. (2015). Basal ganglia system (BGS), bed nucleus of stria terminalis (BNST), pedunculopontine tegmental nucleus, (PPTg), laterodorsal tegmental nucleus (LTDg).

"skeptic" in implementing a Bayesian selection process at the level of individual dopamine cells. Congruence in these two axes, that is, excitatory drive is complemented by selective disinhibition, facilitates increased dopamine cell activity. When this arises across a large percentage of dopamine cells, a product of learning, synchrony emerges facilitating a population based phasic signal.

In our Bayesian construal, the computational goal is to determine the probability, the posterior, that distributed, parallel processes are responding to the same events in the on-going sensorimotor stream (e.g., a cue light predicting reward or the absence of an expected reward), reflecting the ability of those events to broadly organize neural activity, reflecting in turn implicit value; that is, to determine the probability that disparate primary substrates are organizing around and responding to the same events, consensus. Taking the excitatory $(E)$ and inhibitory (I) axes proposed above as advocate and skeptic, respectively, we formulate our Bayesian construal as follows (Fig. 4):

The prior is comprised of the excitatory drive, $P(E)$, reflecting the sampling of distributed processing discussed above. This afferent activity reflects "prior belief" because, as events enter the sensorimotor stream, the response of distributed afferents is shaped by prior experience and learning (response to novel stimuli addressed below). Thus, when multiple afferent regions respond to a sensorimotor event, this increases $\mathrm{P}(\mathrm{E})$, reflecting the prior probability that this event has an organizing impact on distributed processing.

The likelihood, $P(I \mid E) / P(I)$, is a function of striatal inhibition of dopamine. The denominator, $\mathrm{P}(\mathrm{I})$ reflects tonic inhibitory tone; this is the skeptic, maintaining a basal, inhibitory non-belief that diminishes the excitatory prior. This basal inhibitory tone is modulated by afferent input to the striatum (from many of the same regions contributing to the midbrain), $P(|| E)$; that is, striatal inhibitory tone given the excitatory drive on the striatum. Thus, as events in the sensorimotor stream, such as a cue light, disinhibit striatal inhibition of the midbrain, the skeptic is diminished granting greater weight to the prior. We note that the "E" in $P(E)$ and $P(I \mid E)$ reflect the excitatory drives on the midbrain and striatum, respectively, and are not strictly the same quantity. We construe them as serving the same function in our construal, reflecting activity across distributed, parallel processing, i.e., primary processing, funneled to both the striatum and midbrain for different purposes.

We propose that this Bayesian selector operates both at the level of individual cells and the population level. We suggested above that each dopamine cell is sampling afferent activity and serving as an index; here we elaborate this as a Bayesian index. As greater numbers of individual dopamine cells respond to the same events, emergent synchrony will increase, and with it a temporally defined phasic dopamine signal. Thus, the selector operates at the population level simply as a summation across cells, an index of indices, the sum of posterior probabilities from a population of afferent samples.

While we present here apparent mathematical quantities, $P(E)$, etc., these are very different from discrete quantities proposed in prior models, such as expected reward, $V_{t}$. $P(E)$ can be construed as evidentiary without constraining the representational content of the afferent activity. Such activity, as Tian et al. (2016) demonstrated, can reflect value, prediction, prediction errors or, more to the point, activity arising entirely independent of value as an abstract quantity, such as signals reflecting proprioceptive or motor activity, motivational state/choice, arousal and so on. That is, as "evidence in favor of prior 


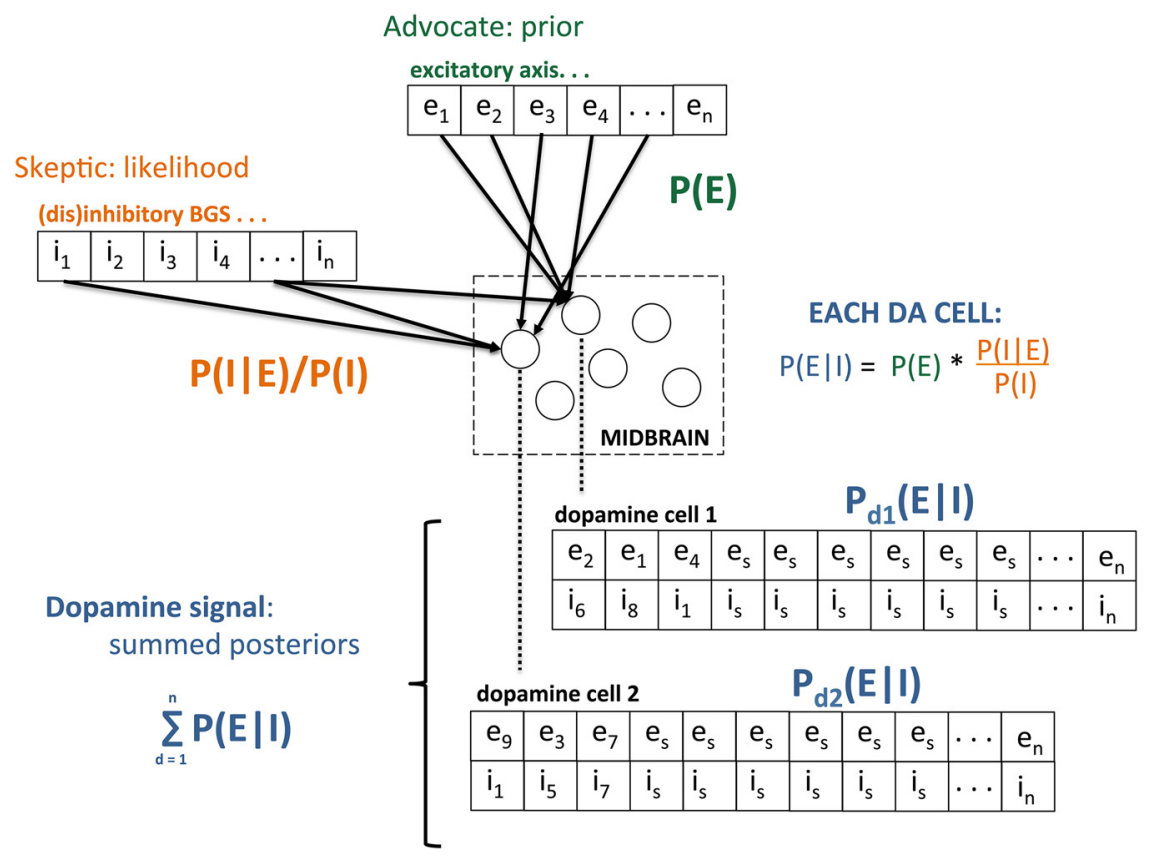

Figure 4. Illustration of midbrain dopamine as a Bayesian selector. Random distribution of vectorized inputs from an afferent input space, as illustrated in Figure 1, where each dopamine cell samples on-going activity, mapped onto a Bayesian construal. The excitatory axis (green) is assigned as the prior (the advocate) and the (dis)inhibitory axis (orange) is assigned as the likelihood (the skeptic). The posterior (blue) arises from the integration of these two axes (i.e., Fig. 3) at both the level of individual dopamine cells (firing rate) and at a population level, where synchrony determines the degree to which increases in firing rates in individual cells sum to produce a population-based phasic signal, which we construe as a consensus index, both consensus across dopamine cells as Bayesian units and consensus across the sampled input space, reflecting widespread afferent activity in response to current stimuli.

belief" the representational content of afferent input can be broad rather than constrained to a single quantitative representation such as value or prediction error. This is an initial conceptual sketch. How such Bayesian operations could be implemented at the cellular level would require further formal theoretical development and empirical testing of the hypothesis (for the challenges inherent in developing neural implementations, see Potjans et al., 2011, 2009).

In sum, we propose that midbrain dopamine is constantly sampling distributed neural activity monitoring for occasions when the input from both the advocate, $P(E)$ and the skeptic, $P(I \mid E) / P(I)$ agree that the current activity distributed across the brain reflects coordinated neural activity in response to an event in the sensorimotor stream that organizes distributed, parallel processing, reflecting implicit value: and as such, likely an opportunity to be acted on and learned about. When a large percentage of dopamine cells arrive at the same "conclusion," synchrony arises and a temporally resolved fluctuation in extracellular dopamine emerges and encodes opportunity to act and learn.

\section{Distributed Cascading Learning: Midbrain Dopamine, the Last to Learn}

The notion that phasic dopamine signals consensus may at first seem contradictory to the widely accepted idea that dopamine provides a teaching signal. In our hypothesis, it appears that midbrain dopamine is the last to learn, not the teacher. This is consistent with studies that observe dopamine prediction errors emerge follow- ing, not preceding learning (Coddington and Dudman, 2018). We will briefly describe a whole-brain learning scheme in which learning occurs in a cascading, interleaved fashion along three axes (Fig. 5).

First, primary learning occurs in afferent regions contributing to midbrain dopamine, such as the PFC, amygdala, hippocampus; that is, across the distributed substrates we called primary processing and identify as the prior, $P(E)$. Second, learning occurs in the striatum and BGS system, altering inhibitory drive on dopamine, the likelihood, $P(I \mid E) / P(I)$. Finally, learning occurs in the midbrain itself via synaptic plasticity, selectively strengthening and weakening particular synaptic inputs. We view this learning as cascading because it occurs in an interleaved fashion where each axis of learning modulates the others. For example, as primary substrates undergo learning, this alters the input driving both the BGS and midbrain, modulating both $P(E)$ and $P(I \mid E)$ in our Bayesian construal. Learning and activity in the primary substrates are, in turn, modulated by both BGS output and dopamine signals. Similarly, learning in the BGS alters inhibitory input to the midbrain, and modulated dopamine in turn alters learning in the striatum. The core idea here is that learning does not occur discretely in one substrate and then get transferred to another as a teaching signal, but that learning occurs simultaneously across all substrates in an interdependent fashion.

A crucial objective for learning in distributed substrates is to coalesce into an organized whole-brain model to 


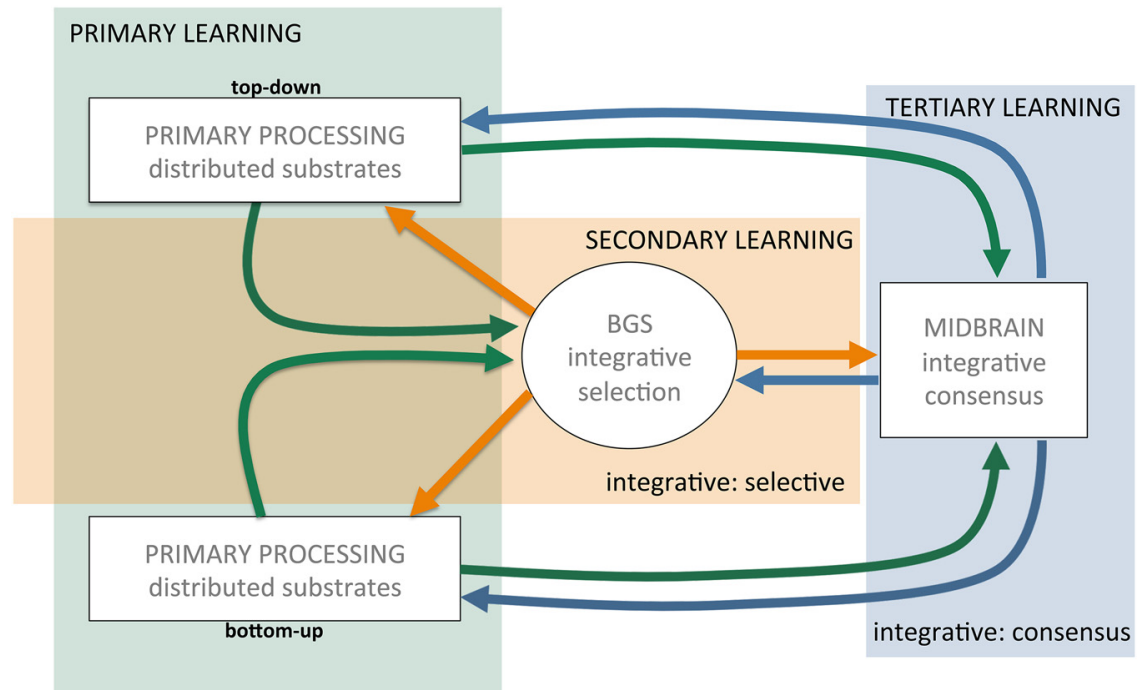

Figure 5. Cascading learning. Learning occurs progressively interleaved at three levels: (1) in primary models of individual afferent substrates, both top-down and bottom-up (blue), (2) in a secondary, integrative selection model in the basal ganglia system (BGS, orange), and (3) in the midbrain dopamine system itself (green). Arrows indicate how learning at different levels influences learning at other levels.

facilitate unitary action. The interleaving of BGS and midbrain learning contributes to the development of this coordinated organization. Midbrain dopamine signals growing consensus as learning progresses, arising through emergent synchrony (Kim et al., 2012), increasing agreement across dopamine cells in their Bayesian computations, which facilitates and accelerates learning as well as mobilizes resources and responding. The ability of midbrain dopamine to modulate learning and responding is effectively titrated by the degree of synchrony that contributes to a temporally resolved phasic signal. In this sense, dopamine has its greatest impact when it is maximally reflecting consensus. That is, the emergence of a phasic dopamine signals reflects the cumulative, convergent effects of cascading learning broadly distributed across the brain, consistent with observations that changes in dopamine signals can lag behind adaptive behavior (Coddington and Dudman, 2018).

This provides an alternative perspective on the emergence of prediction errors. Learning is initiated when an animal encounters known value (e.g., food pellet) at an unexpected place and time. The prediction error hypothesis argues that surprise, the unexpectedness of that initial encounter, generates the dopamine teaching signal. Our hypothesis focuses on the known value aspect of that initial encounter: the encountered food pellet is well known to distributed neural substrates and induces a consistent, organizing response in those substrates that drives a dopamine consensus signal to facilitate further learning about the features of time and place that yielded the food opportunity, such as a cue light predicting the reward. We would further suggest that the emergent phasic dopamine response to the cue light does not reflect dopamine teaching target regions about the value of the cue light but, rather, that the "rest of the brain" has learned the value of the cue light and, as the cue light becomes a stimulus of "known value" across the brain, dopamine now signals consensus regarding the cue light: the last to learn.

\section{Predicted Rewards and Novel Stimuli}

We wish to briefly touch on two possible objections to our notion that dopamine signals consensus. In one, a lack of phasic dopamine can be observed when one might, according to our hypothesis, expect it. In the other, conversely, dopamine cell bursting is observed when there would seem to be no basis for consensus across distributed processing.

First, sometimes a well-predicted reward does not generate a phasic dopamine response. If the reward induces an organized, consistent response across distributed substrates, consensus, why does the dopamine signal disappear? We note first that this is not always the case, perhaps mostly not the case, and that a persistent dopamine signal in response to reward, even in well-learned tasks, has been repeatedly observed (Hamid et al., 2016, and many others). Nevertheless, when the loss of phasic dopamine in response to reward is observed, this arises when a task is overlearned. We would argue that this reflects automation where discrete stimuli (e.g., cueresponse-reward) are "chunked" into a sequence as a single entity (Graybiel, 1998), where only the beginning of the sequence generates a phasic dopamine response, unless the outcome varies from how the sequence is expected to end. That is, the value of the reward is absorbed into the sequence rather than treated as a distinct event. In a sense, this is the same as saying the reward is predicted, only the proposed mechanism is different. Rather than a computation subtracting expected and received reward generating an error of zero when the reward is perfectly predicted, our hypothesis would argue that once a sequence of events (stimuli, actions, outcomes) have become automatized, the individual steps in the sequence no longer organize distributed processing 
nor drive the dopamine response; essentially, they are taken for granted until something goes awry, bringing non-automatic distributed processing back online to process the error, modulating dopamine.

Second, novelty is known to induce increased dopamine cell activity (Horvitz, 2000; Overton et al., 2014), which seems inconsistent with the notion that dopamine is signaling consensus: how can there be consensus across substrates with a newly encountered stimulus or context? Novel sensory information entering the brain does not have a learned path of propagation determined through prior experience; instead, we assume the novel information generates widespread activity across regions as the brain tries to process this new encounter based on prior experience and knowledge, with features of new stimuli partially activating memory of previous stimuli encountered as the brain uses its existing models to learn about the unfamiliar. We would expect this increased activity across distributed brain regions to drive the excitatory axis and generate bursting and small dopamine transients; however, we would not expect synchronous firing phase-locked with stimuli but randomly distributed bursts/transients throughout exploration and examination of novel stimuli. That is, this increased activity does not generate a temporally resolved signal arising from synchronous dopamine activity but instead the bursts/transients are distributed across time, effectively increasing tonic dopamine facilitating increased activity, exploration and plasticity. If new stimuli are repeatedly paired with value, they will come to evoke a clear, temporally resolved phasic signal arising from synchronous activity across a population of dopamine cells. If not, the animal will habituate and tonic dopamine will return to basal levels without acquiring a phasic response. In both cases, our arguments could be elaborated and refined, but space preclude exhaustive discussion.

\section{So, What Does Dopamine Encode?}

Temporal-difference theories of dopamine have been largely based on the idea that dopamine signals arise from a model-free system (Montague et al., 1996). The values associated with a state (e.g., "cue light on") are derived from experience in which the basis of that value is inaccessible, i.e., there is no model of how that value was derived, sometimes called a "cached" value. As a consequence, dopamine provides a "pure" value-related signal in a "common currency" that is independent of distinct sensory features of experience; that is, two rewards of equal value should be substitutable and generate the same dopamine signal. However, it has become increasingly clear that dopamine does not provide a pure, modelfree signal of value (Takahashi et al., 2017; Keiflin et al., 2019); rather, it appears that dopamine signaling may be model-based (Daw et al., 2011; Daw, 2012; Langdon et al., 2018) such that not only changes in value, but the identity and characteristics of sensory information associated with that value can modulate the signal. Moreover, evidence is accumulating that midbrain dopamine may not be restricted solely to value-related signals. As discussed in the introduction, phasic dopamine activity has been shown to correlate to motor movement (Dodson et al., 2016; Howe and Dombeck, 2016), arousal (EbanRothschild et al., 2016), motivational state (Satoh et al., 2003; Syed et al., 2016), and timing (Pasquereau and Turner, 2015; Soares et al., 2016). How do we account for this apparent diversity in the "content" of dopamine signals, i.e., that dopamine does not correlate only to abstract value but can be correlated with a multiplicity of phenomena? Our hypothesis suggests the following.

\section{Primacy of temporal encoding}

We suggest the crucial characteristic of dopamine signaling is when, not what. Dopamine mobilizes and energizes responding and facilitates learning. As a consensus signal, phasic dopamine is effectively saying "now," which serves to activate and coordinate diverse target regions to collectively respond rapidly and vigorously in order to seize upon an opportunity. The crucial question, when is now?, can be answered with varying degrees of temporal precision: from a cue light indicating reward in one second to contextual stimuli, such as being in an task environment with a higher rate of reward availability (Howe et al., 2013; Hamid et al., 2016; Beeler and Mourra, 2018). In our consensus signaling theory, the precision of when is determined by the degree of synchrony among dopamine neurons, such that high synchronous activity leads to temporally resolved phasic signaling while asynchronous activity, even if bursting, leads to increased dopamine distributed across a period of time, i.e., increased tonic, as might occur in a high reward context. That timing is an integral part of dopamine signaling is an established idea, particularly with prediction error theories (Pasquereau and Turner, 2015; Soares et al., 2016; Starkweather et al., 2017; Langdon et al., 2018). Here, we suggest temporal characteristics are more than a feature of dopamine signaling, but its fundamental, primary function: the conductor's baton signaling to distributed processing "this we know, act now."

\section{Bayesian model of opportunity}

The point above begs the question, temporal model of what? We argue the "what" is when an event in the sensorimotor stream impacts neural activity and processing across multiple afferent substrates indicating the event has an organizing effect on distributed, parallel processing, reflecting implicit value. Such implicit value may correspond to the traditional economic sense, such as when a cue light predicts the imminent arrival of a delectable, tasty treat, but may also reflect implicit value in stimuli that organize neural activity to achieve nontraditional value, such as the ability to run the rotarod without falling off, or to execute a killer tennis serve. In essence, it is the neural activity itself that is being assigned a valuation for its utility in achieving some goal rather than valuation of what it is that neural activity is putatively representing. In short, the what corresponds to "when this neural activity arises, it reflects an opportunity for advantage and should be promoted and acted upon." 


\section{Representational content embedded in dopamine signal is unconstrained}

In our view, dopamine cells are collecting evidence, the midbrain is an evidentiary system, and that the representational nature (the content) of that evidence is unconstrained: basically, it can be anything and reflect whatever representational content is being processed in afferent regions driving midbrain dopamine activity. In our metaphor of a dopamine cell as analogous to an index of stocks, if one stock has a disproportionate effect on the index, say an automobile company, this does not mean that the index is "signaling automobile," but if you look at what the index corresponds to in economic activity, it will correspond to increased car sales. Similarly, midbrain dopamine may generate a phasic response, signaling consensus and implicit value, that may correlate with any number of events, stimuli and actions depending upon the task. During a motor task such as the rotarod, dopamine cells may correlate to proprioceptive, vestibular and motor activity. During a task with visual cues, visual information may be embedded in and even dominate dopamine cell activity while in a task with auditory cues, auditory information dominates. In short, rather than encoding an abstracted value associated with different events, stimuli and actions, we propose that these appear in dopamine cell activity precisely to the extent to which they organize distributed, parallel processing in afferent regions, reflecting implicit not abstracted value.

\section{Areas for Further Development and Research}

As noted above, many aspects of our hypothesis are consistent with extant data, including heterogeneity of afferents from distributed brain regions, the requirement for dopamine cells to fire synchronously to generate a temporally resolved phasic signal, evidence that dopamine cell synchrony can increase with learning, evidence that various types of value information are mixed and distributed across multiple afferent substrates, the mixed nature of the dopamine value signals (value, prediction errors), as well as accumulating evidence that dopamine signals can correlate to diverse phenomena beyond abstracted value (i.e., are multifaceted or multiplexed), including both features of stimuli associated with value (model-based signals) as well as "non-value" related activity, such as observed in non-reward related tasks (e.g., motor tasks). What we offer is a different framework in which to interpret these data and an alternative hypothesis on what the "basic function" of dopamine might be, shifting from signaling and teaching about reward or value to mediating coordination across distributed, parallel processing.

This initial description of our hypothesis, laying out the basic ideas and claims, lacks the rigor of a formal theory, which will emerge over time as the ideas presented are further developed. Nonetheless, the hypothesis does yield many predictions that can be tested empirically. For example, the notion that "dopamine is the last to learn" can be tested in a design similar to Tian et al. (2016), except looking at the progression of the mixed value signals in afferents compared to midbrain dopamine across learning. Similarly, optical tools can facilitate comparing inhibitory striatal inputs, $P(|| E) / P(I)$, to excitatory inputs, $P(E)$ to begin to empirically test and dissect our Bayesian formulation. Readers can discern such testable predictions themselves. Given the nascent nature of our hypothesis, we use available space here to note some limitations in our initial discussion and areas for further development, consideration and research.

\section{Limitations}

First, it is becoming increasingly evident that both dopamine and midbrain GABA neurons form a functional midbrain unit (Brown et al., 2012; van Zessen et al., 2012), including the recent hypothesis that the dopamine prediction error arises as a subtractive computation between GABA and dopamine cells in the midbrain (Eshel et al., 2015). A more comprehensive hypothesis of "midbrain dopamine" may require inclusion of GABA neurons. Second, we have glossed over the extent to which midbrain dopamine may consist of functionally separate subpopulations that signal independently, possibly through different "channels" with distinct, segregated projection targets (Willuhn et al., 2012; Roeper, 2013; Lammel et al., 2014; Sanchez-Catalan et al., 2014; Dreyer et al., 2016; Morales and Margolis, 2017). Third, our hypothesis would naturally lead to the question of a role for synchronized oscillations at various frequencies between the midbrain and other brain regions. Dreyer et al. (2016) demonstrate that cocaine induces $0.5-\mathrm{Hz}$ oscillations in dopamine release in the nucleus accumbens. Fujisawa and Buzsáki (2011) have demonstrated $4-\mathrm{Hz}$ oscillatory activity in the VTA that couples with prefrontal oscillations. The authors suggest that midbrain dopamine may play a role in synchronizing this oscillatory activity across brain regions. Such oscillatory activity in the midbrain has been surprisingly little studied and could be introduced into the current hypothesis. Fourth, dopamine cells release multiple transmitters (Trudeau et al., 2014), including glutamate, GABA and sonic hedgehog (Gonzalez-Reyes et al., 2012), not addressed here. Our hypothesis is built around dopamine volume transmission. What role intrasynaptic neurotransmission at dopamine synapses may play is not clear. Interestingly, Fujisawa and Buzsáki (2011) suggest that glutamate release from dopamine terminals may play a role in regulating oscillatory synchronization. Although not incorporated into our hypothesis, this notion is certainly consistent with midbrain dopamine serving a role in coordinating distributed, parallel processing.

\section{Areas for development}

Our hypothesis posits that dopamine cells comprise a layer in cascading learning. While dopamine cells exhibit synaptic plasticity, this plasticity and its regulation has not been as extensively characterized as, for example, corticostriatal plasticity. We suggest here that synaptic plasticity in dopamine cells is regulated by inhibitory GABA inputs from the striatum, analogous to how dopamine regulates corticostriatal plasticity. In this way, selective activity-dependent long-term potentiation of excitatory synapses would be gated by disinhibition of striatal in- 
puts. Although there is evidence for this notion (Tan et al, 2010), it has not been exhaustively investigated and characterized. Synaptic plasticity within the midbrain has not, in general, figured prominently into theories of dopamine; knowledge of its mechanisms, regulation and function remain limited and underexplored. While dopamine has been suggested to provide an instructional signal to the striatum, we suggest that the striatum in turn provides an instructional signal to the midbrain. In this construal, this striatal teaching signal, the likelihood, gates plasticity at excitatory synapses, modifying transmission of excitatory drive, the prior, from primary processing, in future encounters.

Another area for exploration is how different inputs are actually integrated at a cellular level. Our Bayesian construal would on first glance suggest that inhibitory inputs from the striatum and excitatory inputs from distributed afferents should be multiplicative, but this is not exactly right. The likelihood, $\mathrm{P}(\mathrm{I} \mid \mathrm{E}) / \mathrm{P}(\mathrm{I})$ is not computed in the midbrain where excitatory activity, $\mathrm{P}(\mathrm{E})$ is multiplied by $\mathrm{P}(\mathrm{I}$ I E) and both quantities divided by $P(I)$. Rather, in our construal, the likelihood is computed in the striatum and delivered as a single quantity of disinhibition to the midbrain; mathematically, $P(E)$ is not divided directly by $P(I)$. The question might be whether $P(E)+P(I \mid E) / P(I)$, likely a more accurate rendering, is functionally equivalent. Using cortical activity as an example, if specific cortical activity (say that encoding a cue light) contributes afferent drive to both midbrain, i.e., $P(E)$ and the striatum, i.e., $P(|| E)$, could an increase in this cortical activity transmitted to the midbrain both directly and via disinhibition be multiplicative in the degree to which that specific cortical activity drives midbrain dopamine? The answer is unknown. Demonstrating how mathematical operations comprising formal theories are implemented in neural machinery is a continuing challenge (Potjans et al., 2009, 2011).

Finally, our initial description of the hypothesis provided here requires more formal theoretical elaboration. However, doing so entails rethinking what a normative description of dopamine function might mean if its primary role is construed as mediating coordination across distributed processing rather than signaling value per se. As long as dopamine is viewed in some fashion as the reward transmitter, computational approaches can build formal algorithms whose functional goal is maximizing value. Our hypothesis posits "maximizing value" as a distal goal mediated by multiple neural substrates where the proximal problem is getting those substrates to function in a coordinated manner to achieve maximal value. If dopamine is construed as a solution to that proximal problem of coordination, then thinking through formal models of this idea requires developing notions on how "coordination" is maximized or even quantified and evaluated. We believe our Bayesian construal of dopamine and notion of cascading, distributed learning offers some initial fodder for more formal efforts.

\section{Conclusion}

A good hypothesis should provoke novel investigations and generate deeper understanding, whether ultimately proven true or not. The prediction error hypothesis makes clear, simple predictions: that phasic dopamine signaling will correspond to discrepancies in expected and actual outcomes. Nonetheless, twenty years later we are still testing this hypothesis (Watabe-Uchida et al., 2017), which has proven to be a rich driver of experimental investigation giving rise to a deeper, more complex understanding of midbrain dopamine (Eshel et al., 2015; Hamid et al., 2016; Langdon et al., 2018), although the extent to which it is correct continues to be subject to debate (Berke, 2018).

Various theories of dopamine function have been proposed. Data have accumulated supporting each of these theories and, strictly speaking, falsifying each other. If a value instead of error signal is observed, then strictly speaking the prediction error hypothesis cannot be a complete account. The taste for pitting these different accounts of dopamine against each other seems to be waning, with a growing appreciation that each likely captures some aspect of dopamine signaling. Increasingly, the most pressing question seems to be how to conceive of a framework where these different theories can be integrated rather than viewed as competing accounts.

By shifting from a presupposition that dopamine fundamentally signals value information in some fashion, the reward transmitter, to positing that dopamine plays a role in mediating coordination across distributed, parallel processing, the hypothesis outlined here provides an alternative perspective in thinking about how to assimilate and interpret the diverse data that have accumulated for decades on dopamine. Moreover, it suggests new avenues for both theoretical and empirical exploration and model development. This is particularly true in light of data emerging in recent years showing much greater richness and complexity to dopamine signals, as well as its connectivity, architecture and physiology, than originally imagined. As it becomes increasingly difficult to shoehorn this apparent complexity into one or another extant theories, the need for alternative frameworks, perspectives and accounts may grow. Although only an initial description, we offer the current hypothesis in the spirit of this need for new perspectives.

\section{References}

Agnati LF, Zoli M, Strömberg I, Fuxe K (1995) Intercellular communication in the brain: wiring versus volume transmission. Neuroscience 69:711-726.

Alexander GE (1994) Basal ganglia-thalamocortical circuits: their role in control of movements. J Clin Neurophysiol 11:420-431.

Arbuthnott GW, Wickens J (2007) Space, time and dopamine. Trends Neurosci 30:62-69.

Bar-Gad I, Morris G, Bergman H (2003) Information processing, dimensionality reduction and reinforcement learning in the basal ganglia. Prog Neurobiol 71:439-473.

Barto AG (1995) Adaptive critic and the basal ganglia. In: Models of Information Processing in the Basal Ganglia, pp 215-232. Cambridge, MA: MIT Press.

Beeler JA, Mourra D (2018) To do or not to do: dopamine, affordability and the economics of opportunity. Front Integr Neurosci 12:6.

Beier KT, Steinberg EE, DeLoach KE, Xie S, Miyamichi K, Schwarz L, Gao XJ, Kremer EJ, Malenka RC, Luo L (2015) Circuit architecture 
of VTA dopamine neurons revealed by systematic input-output mapping. Cell 162:622-634.

Berke JD (2018) What does dopamine mean? Nat Neurosci 21:787793.

Berridge KC (2007) The debate over dopamine's role in reward: the case for incentive salience. Psychopharmacology (Berl) 191:391431.

Beyeler A, Chang C-J, Silvestre M, Lévêque C, Namburi P, Wildes $\mathrm{CP}$, Tye KM (2018) Organization of valence-encoding and projection-defined neurons in the basolateral amygdala. Cell Rep 22:905-918.

Björklund A, Dunnett SB (2007) Dopamine neuron systems in the brain: an update. Trends Neurosci 30:194-202.

Bolam JP, Pissadaki EK (2012) Living on the edge with too many mouths to feed: why dopamine neurons die. Mov Disord 27:14781483.

Breakspear M (2017) Dynamic models of large-scale brain activity. Nat Neurosci 20:340-352.

Bromberg-Martin ES, Matsumoto M, Hikosaka O (2010) Dopamine in motivational control: rewarding, aversive, and alerting. Neuron 68:815-834.

Brown J, Bullock D, Grossberg S (1999) How the basal ganglia use parallel excitatory and inhibitory learning pathways to selectively respond to unexpected rewarding cues. J Neurosci 19:1050210511.

Brown MTC, Tan KR, O'Connor EC, Nikonenko I, Muller D, Lüscher C (2012) Ventral tegmental area GABA projections pause accumbal cholinergic interneurons to enhance associative learning. $\mathrm{Na}-$ ture 492:452-456.

Buneo CA, Andersen RA (2006) The posterior parietal cortex: sensorimotor interface for the planning and online control of visually guided movements. Neuropsychologia 44:2594-2606.

Carta I, Chen CH, Schott AL, Dorizan S, Khodakhah K (2019) Cerebellar modulation of the reward circuitry and social behavior. Science 363:eaav0581.

Choi EY, Tanimura Y, Vage PR, Yates EH, Haber SN (2017) Convergence of prefrontal and parietal anatomical projections in a connectional hub in the striatum. Neuroimage 146:821-832.

Christophel TB, Klink PC, Spitzer B, Roelfsema PR, Haynes J-D (2017) The distributed nature of working memory. Trends Cogn Sci 21:111-124

Cisek P, Kalaska JF (2010) Neural mechanisms for interacting with a world full of action choices. Annu Rev Neurosci 33:269-298.

Coddington LT, Dudman JT (2018) The timing of action determines reward prediction signals in identified midbrain dopamine neurons. Nat Neurosci 21:1563-1573.

Contreras-Vidal JL, Schultz W (1999) A predictive reinforcement model of dopamine neurons for learning approach behavior. $\mathrm{J}$ Comput Neurosci 6:191-214.

Cragg SJ, Rice ME (2004) Dancing past the DAT at a DA synapse. Trends Neurosci 27:270-277.

Cui H (2014) From intention to action: hierarchical sensorimotor transformation in the posterior parietal cortex. eNeuro 1: ENEURO.0017-14.2014.

Da Cunha C, Wietzikoski EC, Dombrowski P, Bortolanza M, Santos LM, Boschen SL, Miyoshi E (2009) Learning processing in the basal ganglia: a mosaic of broken mirrors. Behav Brain Res 199: 157-170.

da Silva JA, Tecuapetla F, Paixão V, Costa RM (2018) Dopamine neuron activity before action initiation gates and invigorates future movements. Nature 554:244-248.

Daw ND (2012) Model-based reinforcement learning as cognitive search: neurocomputational theories. In: Cognitive Search: Evolution, Algorithms, and the Brain (Todd PM, Hills TT, Robins TW, eds), pp 195-207. Cambridge: IEEE; MIT Press.

Daw ND, Gershman SJ, Seymour B, Dayan P, Dolan RJ (2011) Model-based influences on humans' choices and striatal prediction errors. Neuron 69:1204-1215.
Day JJ, Wheeler RA, Roitman MF, Carelli RM (2006) Nucleus accumbens neurons encode Pavlovian approach behaviors: evidence from an autoshaping paradigm. Eur J Neurosci 23:1341-1351.

Day JJ, Jones JL, Carelli RM (2011) Nucleus accumbens neurons encode predicted and ongoing reward costs in rats: nucleus accumbens and reward cost. Eur J Neurosci 33:308-321.

den Ouden HEM, Daunizeau J, Roiser J, Friston KJ, Stephan KE (2010) Striatal prediction error modulates cortical coupling. J Neurosci 30:3210-3219.

Dodson PD, Dreyer JK, Jennings KA, Syed ECJ, Wade-Martins R, Cragg SJ, Bolam JP, Magill PJ (2016) Representation of spontaneous movement by dopaminergic neurons is cell-type selective and disrupted in parkinsonism. Proc Natl Acad Sci USA 113: E2180-E2188.

Dreyer JK (2014) Three mechanisms by which striatal denervation causes breakdown of dopamine signaling. J Neurosci 34:1244412456.

Dreyer JK, Hounsgaard J (2013) Mathematical model of dopamine autoreceptors and uptake inhibitors and their influence on tonic and phasic dopamine signaling. J Neurophysiol 109:171-182.

Dreyer JK, Herrik KF, Berg RW, Hounsgaard JD (2010) Influence of phasic and tonic dopamine release on receptor activation. J Neurosci 30:14273-14283.

Dreyer JK, Vander Weele CM, Lovic V, Aragona BJ (2016) Functionally distinct dopamine signals in nucleus accumbens core and shell in the freely moving rat. J Neurosci 36:98-112.

Eban-Rothschild A, Rothschild G, Giardino WJ, Jones JR, de Lecea $L$ (2016) VTA dopaminergic neurons regulate ethologically relevant sleep-wake behaviors. Nat Neurosci 19:1356-1366.

Eshel N, Bukwich M, Rao V, Hemmelder V, Tian J, Uchida N (2015) Arithmetic and local circuitry underlying dopamine prediction errors. Nature 525:243-246.

Eshel N, Tian J, Bukwich M, Uchida N (2016) Dopamine neurons share common response function for reward prediction error. Nat Neurosci 19:479-486.

Fujisawa S, Buzsáki G (2011) A 4 Hz oscillation adaptively synchronizes prefrontal, VTA, and hippocampal activities. Neuron 72:153165.

Garris PA, Ciolkowski EL, Pastore P, Wightman RM (1994) Efflux of dopamine from the synaptic cleft in the nucleus accumbens of the rat brain. J Neurosci 14:6084-6093.

Gauthier JL, Tank DW (2018) A Dedicated Population for Reward Coding in the Hippocampus. Neuron 99:179-193.e7.

German PW, Fields HL (2007) Rat nucleus accumbens neurons persistently encode locations associated with morphine reward. J Neurophysiol 97:2094-2106.

Gerraty RT, Davidow JY, Foerde K, Galvan A, Bassett DS, Shohamy D (2018) Dynamic flexibility in striatal-cortical circuits supports reinforcement learning. J Neurosci 38:2442-2453.

Gonon F (1997) Prolonged and extrasynaptic excitatory action of dopamine mediated by $\mathrm{D} 1$ receptors in the rat striatum in vivo. $\mathrm{J}$ Neurosci 17:5972-5978.

Gonzalez-Reyes LE, Verbitsky M, Blesa J, Jackson-Lewis V, Paredes D, Tillack K, Phani S, Kramer ER, Przedborski S, Kottmann AH (2012) Sonic hedgehog maintains cellular and neurochemical homeostasis in the adult nigrostriatal circuit. Neuron 75:306-319.

Grace AA, Bunney BS (1983) Intracellular and extracellular electrophysiology of nigral dopaminergic neurons-3. Evidence for electrotonic coupling. Neuroscience 10:333-348.

Graybiel AM (1998) The basal ganglia and chunking of action repertoires. Neurobiol Learn Mem 70:119-136.

Guo Q, Wang D, He X, Feng Q, Lin R, Xu F, Fu L, Luo M (2015) Whole-brain mapping of inputs to projection neurons and cholinergic interneurons in the dorsal striatum. PLoS One 10:e0123381.

Haber SN, Fudge JL, McFarland NR (2000) Striatonigrostriatal pathways in primates form an ascending spiral from the shell to the dorsolateral striatum. J Neurosci 20:2369-2382.

Hamid AA, Pettibone JR, Mabrouk OS, Hetrick VL, Schmidt R, Vander Weele CM, Kennedy RT, Aragona BJ, Berke JD (2016) 
Mesolimbic dopamine signals the value of work. Nat Neurosci 19:117-126.

Hart AS, Rutledge RB, Glimcher PW, Phillips PEM (2014) Phasic dopamine release in the rat nucleus accumbens symmetrically encodes a reward prediction error term. J Neurosci 34:698-704.

Haruno M, Kawato M (2006) Heterarchical reinforcement-learning model for integration of multiple cortico-striatal loops: fMRI examination in stimulus-action-reward association learning. Neural Netw 19:1242-1254.

Hazy TE, Frank MJ, O'Reilly RC (2007) Towards an executive without a homunculus: computational models of the prefrontal cortex/ basal ganglia system. Philos Trans R Soc B Biol Sci 362:16011613.

Hazy TE, Frank MJ, O'Reilly RC (2010) Neural mechanisms of acquired phasic dopamine responses in learning. Neurosci Biobehav Rev 34:701-720.

Hikosaka O, Sakamoto M, Usui S (1989a) Functional properties of monkey caudate neurons. I. Activities related to saccadic eye movements. J Neurophysiol 61:780-798.

Hikosaka O, Sakamoto M, Usui S (1989b) Functional properties of monkey caudate neurons. II. Visual and auditory responses. J Neurophysiol 61:799-813.

Hikosaka O, Sakamoto M, Usui S (1989c) Functional properties of monkey caudate neurons. III. Activities related to expectation of target and reward. J Neurophysiol 61:814-832.

Hikosaka O, Kim HF, Yasuda M, Yamamoto S (2014) Basal ganglia circuits for reward value-guided behavior. Annual Review of Neuroscience 37:289-306.

Horga G, Maia TV, Marsh R, Hao X, Xu D, Duan Y, Tau GZ, Graniello B, Wang Z, Kangarlu A, Martinez D, Packard MG, Peterson BS (2015) Changes in corticostriatal connectivity during reinforcement learning in humans: corticostriatal connectivity during learning. Hum Brain Mapp 36:793-803.

Horvitz JC (2000) Mesolimbocortical and nigrostriatal dopamine responses to salient non- reward events. Neuroscience 96:651-656.

Houk JC, Adams JL, Barto AG (1995) A model of how the basal ganglia generate and use neural signals that predict reinforcement. In: Models of information processing in the basal ganglia, pp 249-270. Cambridge, MA: MIT Press.

Howe MW, Dombeck DA (2016) Rapid signalling in distinct dopaminergic axons during locomotion and reward. Nature 535:505-510.

Howe MW, Tierney PL, Sandberg SG, Phillips PEM, Graybiel AM (2013) Prolonged dopamine signalling in striatum signals proximity and value of distant rewards. Nature 500:575-579.

Humphries MD, Prescott TJ (2010) The ventral basal ganglia, a selection mechanism at the crossroads of space, strategy, and reward. Prog Neurobiol 90:385-417.

Hyland BI, Reynolds JNJ, Hay J, Perk CG, Miller R (2002) Firing modes of midbrain dopamine cells in the freely moving rat. Neuroscience 114:475-492.

Joel D, Niv Y, Ruppin E (2002) Actor-critic models of the basal ganglia: new anatomical and computational perspectives. Neural Netw 15:535-547.

Joshua M, Adler A, Prut Y, Vaadia E, Wickens JR, Bergman H (2009) Synchronization of midbrain dopaminergic neurons is enhanced by rewarding events. Neuron 62:695-704.

Chang JY, Chen L, Luo F, Shi LH, Woodward DJ (2002) Neuronal responses in the frontal cortico-basal ganglia system during delayed matching-to-sample task: ensemble recording in freely moving rats. Exp Brain Res 142:67-80.

Kakade S, Dayan P (2002) Dopamine: generalization and bonuses. Neural Netw 15:549-559.

Kawato M, Samejima K (2007) Efficient reinforcement learning: computational theories, neuroscience and robotics. Curr Opin Neurobiol 17:205-212.

Kemp JM, Powell TP (1971) The connexions of the striatum and globus pallidus: synthesis and speculation. Trans $\mathrm{R}$ Soc Lond $\mathrm{B}$ Biol Sci 262:441-457.
Keiflin R, Pribut HJ, Shah NB, Janak PH (2019) Ventral tegmental dopamine neurons participate in reward identity predictions. Curr Biol 29:93-103.e3.

Kim Y, Wood J, Moghaddam B (2012) Coordinated activity of ventral tegmental neurons adapts to appetitive and aversive learning. PLoS One 7:e29766.

Kishida KT, Saez I, Lohrenz T, Witcher MR, Laxton AW, Tatter SB, White JP, Ellis TL, Phillips PEM, Montague PR (2016) Subsecond dopamine fluctuations in human striatum encode superposed error signals about actual and counterfactual reward. Proc Natl Acad Sci USA 113:200-205.

Ko D, Wanat MJ (2016) Phasic dopamine transmission reflects initiation vigor and exerted effort in an action- and region-specific manner. J Neurosci 36:2202-2211.

Komendantov AO, Canavier CC (2002) Electrical coupling between model midbrain dopamine neurons: effects on firing pattern and synchrony. J Neurophysiol 87:1526-1541.

Lammel S, Lim BK, Malenka RC (2014) Reward and aversion in a heterogeneous midbrain dopamine system. Neuropharmacology 76:351-359.

Langdon AJ, Sharpe MJ, Schoenbaum G, Niv Y (2018) Model-based predictions for dopamine. Curr Opin Neurobiol 49:1-7.

Lerner TN, Shilyansky C, Davidson TJ, Evans KE, Beier KT, Zalocusky KA, Crow AK, Malenka RC, Luo L, Tomer R, Deisseroth K (2015) Intact-brain analyses reveal distinct information carried by SNc dopamine subcircuits. Cell 162:635-647.

Li W, Doyon WM, Dani JA (2011) Acute in vivo nicotine administration enhances synchrony among dopamine neurons. Biochem Pharmacol 82:977-983.

Mamad O, Stumpp L, McNamara HM, Ramakrishnan C, Deisseroth K, Reilly RB, Tsanov M (2017) Place field assembly distribution encodes preferred locations. PLoS Biol 15:e2002365.

Marinelli M, McCutcheon JE (2014) Heterogeneity of dopamine neuron activity across traits and states. Neuroscience 282:176-197.

Matsuda W, Furuta T, Nakamura KC, Hioki H, Fujiyama F, Arai R, Kaneko T (2009) Single nigrostriatal dopaminergic neurons form widely spread and highly dense axonal arborizations in the neostriatum. J Neurosci 29:444-453.

Matsumoto M, Hikosaka O (2009) Two types of dopamine neuron distinctly convey positive and negative motivational signals. Nature 459:837-841.

Montague PR, Dayan P, Sejnowski TJ (1996) A framework for mesencephalic dopamine systems based on predictive hebbian learning. J Neurosci 16:1936-1947.

Morales M, Margolis EB (2017) Ventral tegmental area: cellular heterogeneity, connectivity and behaviour. Nat Rev Neurosci 18:7385

Morris G, Arkadir D, Nevet A, Vaadia E, Bergman H (2004) Coincident but distinct messages of midbrain dopamine and striatal tonically active neurons. Neuron 43:133-143.

Moss J, Bolam JP (2008) A dopaminergic axon lattice in the striatum and its relationship with cortical and thalamic terminals. J Neurosci 28:11221-11230.

Nicola SM, Yun IA, Wakabayashi KT, Fields HL (2004) Cue-evoked firing of nucleus accumbens neurons encodes motivational significance during a discriminative stimulus task. J Neurophysiol 91: 1840-1865.

O'Neill PK, Gore F, Salzman CD (2018) Basolateral amygdala circuitry in positive and negative valence. Curr Opin Neurobiol 49: 175-183.

Overton PG, Vautrelle N, Redgrave P (2014) Sensory regulation of dopaminergic cell activity: phenomenology, circuitry and function. Neuroscience 282:1-12.

Owesson-White CA, Roitman MF, Sombers LA, Belle AM, Keithley RB, Peele JL, Carelli RM, Wightman RM (2012) Sources contributing to the average extracellular concentration of dopamine in the nucleus accumbens: extracellular dopamine concentration. J Neurochem 121:252-262.

Parker NF, Cameron CM, Taliaferro JP, Lee J, Choi JY, Davidson TJ, Daw ND, Witten IB (2016) Reward and choice encoding in termi- 
nals of midbrain dopamine neurons depends on striatal target. Nat Neurosci 19:845-854.

Pasquereau B, Turner RS (2015) Dopamine neurons encode errors in predicting movement trigger occurrence. J Neurophysiol 113: $1110-1123$

Potjans W, Morrison A, Diesmann M (2009) A spiking neural network model of an actor-critic learning agent. Neural Comput 21:301339.

Potjans W, Diesmann M, Morrison A (2011) An imperfect dopaminergic error signal can drive temporal-difference learning. PLoS Comput Biol 7:e1001133.

Pryce CR (2018) Comparative evidence for the importance of the amygdala in regulating reward salience. Curr Opin Behav Sci 22:76-81.

Roeper J (2013) Dissecting the diversity of midbrain dopamine neurons. Trends Neurosci 36:336-342.

Roitman MF (2004) Dopamine operates as a subsecond modulator of food seeking. J Neurosci 24:1265-1271.

Salamone JD, Correa M (2012) The mysterious motivational functions of mesolimbic dopamine. Neuron 76:470-485.

Sanchez-Catalan MJ, Kaufling J, Georges F, Veinante P, Barrot M (2014) The antero-posterior heterogeneity of the ventral tegmental area. Neuroscience 282:198-216.

Satoh T, Nakai S, Sato T, Kimura M (2003) Correlated coding of motivation and outcome of decision by dopamine neurons. J Neurosci 23:9913-9923.

Saunders BT, Richard JM, Margolis EB, Janak PH (2018) Dopamine neurons create Pavlovian conditioned stimuli with circuit-defined motivational properties. Nat Neurosci 21:1072-1083.

Schultz W (1998) Predictive reward signal of dopamine neurons. J Neurophysiol 80:1-27.

Schultz W (2007) Behavioral dopamine signals. Trends Neurosci 30:203-210.

Schultz W, Dayan P, Montague PR (1997) A neural substrate of prediction and reward. Science 275:1593-1599.

Schultz W, Stauffer WR, Lak A (2017) The phasic dopamine signal maturing: from reward via behavioural activation to formal economic utility. Curr Opin Neurobiol 43:139-148.

Sharpe MJ, Chang CY, Liu MA, Batchelor HM, Mueller LE, Jones JL, Niv Y, Schoenbaum G (2017) Dopamine transients are sufficient and necessary for acquisition of model-based associations. Nat Neurosci 20:735-742.

Shibata R, Mulder A, Trullier O, Wiener S (2001) Position sensitivity in phasically discharging nucleus accumbens neurons of rats alternating between tasks requiring complementary types of spatial cues. Neuroscience 108:391-411.

Soares S, Atallah BV, Paton JJ (2016) Midbrain dopamine neurons control judgment of time. Science 354:1273-1277.

Stachenfeld KL, Botvinick MM, Gershman SJ (2017) The hippocampus as a predictive map. Nat Neurosci 20:1643-1653.

Starkweather CK, Babayan BM, Uchida N, Gershman SJ (2017) Dopamine reward prediction errors reflect hidden-state inference across time. Nat Neurosci 20:581-589.

Steinberg EE, Keiflin R, Boivin JR, Witten IB, Deisseroth K, Janak PH (2013) A causal link between prediction errors, dopamine neurons and learning. Nat Neurosci 16:966-973.

Syed ECJ, Grima LL, Magill PJ, Bogacz R, Brown P, Walton ME (2016) Action initiation shapes mesolimbic dopamine encoding of future rewards. Nat Neurosci 19:34-36.

Taha SA, Nicola SM, Fields HL (2007) Cue-evoked encoding of movement planning and execution in the rat nucleus accumbens. J Physiol 584:801-818.

Takahashi YK, Langdon AJ, Niv Y, Schoenbaum G (2016) Temporal specificity of reward prediction errors signaled by putative dopamine neurons in rat VTA depends on ventral striatum. Neuron 91:182-193.
Takahashi YK, Batchelor HM, Liu B, Khanna A, Morales M, Schoenbaum G (2017) Dopamine neurons respond to errors in the prediction of sensory features of expected rewards. Neuron 95: 1395-1405.e3.

Takiyama K (2015) Sensorimotor transformation via sparse coding. Sci Rep 5:9648.

Tan CO, Bullock D (2008) A local circuit model of learned striatal and dopamine cell responses under probabilistic schedules of reward. J Neurosci 28:10062-10074.

Tan KR, Brown M, Labouèbe G, Yvon C, Creton C, Fritschy J-M, Rudolph U, Lüscher C (2010) Neural bases for addictive properties of benzodiazepines. Nature 463:769-774.

Tepper JM, Abercrombie ED, Bolam JP (2007) Basal ganglia macrocircuits. Prog Brain Res 160:3-7.

Tian J, Huang R, Cohen JY, Osakada F, Kobak D, Machens CK, Callaway EM, Uchida N, Watabe-Uchida M (2016) Distributed and mixed information in monosynaptic inputs to dopamine neurons. Neuron 91:1374-1389.

Tremblay L, Hollerman JR, Schultz W (1998) Modifications of reward expectation-related neuronal activity during learning in primate striatum. J Neurophysiol 80:964-977.

Trudeau LE, Hnasko TS, Wallén-Mackenzie Å, Morales M, Rayport S, Sulzer D (2014) The multilingual nature of dopamine neurons. Prog Brain Res 211:141-164.

van Schouwenburg MR, den Ouden HEM, Cools R (2010) The human basal ganglia modulate frontal-posterior connectivity during attention shifting. J Neurosci 30:9910-9918.

Vandecasteele M (2005) Electrical synapses between dopaminergic neurons of the substantia nigra pars compacta. J Neurosci 25 291-298.

van Zessen R, Phillips JL, Budygin EA, Stuber GD (2012) Activation of VTA GABA neurons disrupts reward consumption. Neuron 73: $1184-1194$

Venton BJ, Zhang H, Garris PA, Phillips PEM, Sulzer D, Wightman RM (2003) Real-time decoding of dopamine concentration changes in the caudate-putamen during tonic and phasic firing. $J$ Neurochem 87:1284-1295.

Vitay J, Hamker FH (2014) Timing and expectation of reward: a neuro-computational model of the afferents to the ventral tegmental area. Front Neurorobot 8:4.

Watabe-Uchida M, Zhu L, Ogawa SK, Vamanrao A, Uchida N (2012) Whole-brain mapping of direct inputs to midbrain dopamine neurons. Neuron 74:858-873.

Watabe-Uchida M, Eshel N, Uchida N (2017) Neural circuitry of reward prediction error. Annu Rev Neurosci 40:373-394.

Whitlock JR (2014) Navigating actions through the rodent parietal cortex. Front Hum Neurosci 8:293.

Willuhn I, Burgeno LM, Everitt BJ, Phillips PE (2012) Hierarchical recruitment of phasic dopamine signaling in the striatum during the progression of cocaine use. Proc Natl Acad Sci USA 109:2070320708.

Wilson CJ, Young SJ, Groves PM (1977) Statistical properties of neuronal spike trains in the substantia nigra: cell types and their interactions. Brain Res 136:243-260.

Wise RA (2004) Dopamine, learning and motivation. Nat Rev Neurosci 5:483-494.

Yetnikoff L, Lavezzi HN, Reichard RA, Zahm DS (2014) An update on the connections of the ventral mesencephalic dopaminergic complex. Neuroscience 282:23-48.

Zheng T, Wilson CJ (2002) Corticostriatal combinatorics: the implications of corticostriatal axonal arborizations. J Neurophysiol 87: 1007-1017.

Zoli M, Torri C, Ferrari R, Jansson A, Zini I, Fuxe K, Agnati LF (1998) The emergence of the volume transmission concept. Brain Res Rev 26:136-147. 